\title{
ABC1 Consensus Conference - a German Perspective
}

\author{
First International Consensus Conference on Advanced Breast Cancer (ABC1), Lisbon, \\ November 5, 2011
}

\author{
Christoph Thomssen*a Norbert Marschner*c Michael Untch*d
}

Thomas Decker ${ }^{\mathrm{e}}$ Susanna Hegewisch-Becker ${ }^{f} \quad$ Christian Jackisch $^{g}$ Wolfgang Janni ${ }^{\text {h }}$ Hans-Joachim Lücki Gunter von Minckwitz ${ }^{j}$ Anton Scharl ${ }^{k}$ Andreas Schneeweiss' Hans Tesch ${ }^{m}$ Anja Welt ${ }^{n}$ Nadia Harbeck ${ }^{* b}$

\begin{abstract}
aUniversitätsklinik und Poliklinik für Gynäkologie, Martin-Luther-Universität, Halle/Saale, ${ }^{b}$ Brustzentrum der Ludwig-Maximilians-Universität Universität München,

${ }^{\circ}$ Gemeinschaftspraxis für interdisziplinäre Onkologie und Hämatologie, Freiburg i.Br., ${ }^{d}$ Klinik für Gynäkologie und Geburtshilfe, HELIOS Klinikum Berlin Buch, ${ }^{e}$ Gemeinschaftspraxis Onkologie Ravensburg, ${ }^{f}$ Internistische Praxisgemeinschaft Eppendorf, Hamburg, ${ }^{9}$ Klinik für Gynäkologie und Geburtshilfe, Offenbach, hFrauenklinik des Universitätsklinikums Düsseldorf, 'Gynäkologisch-onkologische Praxis, Hannover, 'GBG Forschungs GmbH, Neu-Isenburg,

${ }^{k}$ Frauenklinik des Klinikums St. Marien, Amberg, 'Nationales Zentrum für Tumorerkrankungen, Sektion Gynäkologische Onkologie, Heidelberg, ${ }^{m}$ Onkologische Gemeinschaftspraxis, Frankfurt, "Westdeutsches Tumorzentrum des Universitätsklinikums Essen, Germany
\end{abstract}

\section{Keywords}

ABC1-consensus - Metastatic breast cancer, diagnosis and staging, treatment - Tumor markers - Metastases, biopsy · Chemotherapy - Endocrine therapy · Anti-HER2targeted therapy - Palliative care

\section{Summary}

A group of German breast cancer experts (medical oncologists and gynaecologists) reviewed and commented on the results of the first international 'Advanced Breast Cancer First Consensus Conference' (ABC1) for the diagnosis and treatment of advanced breast cancer. The $A B C 1$ Conference is an initiative of the European School of Oncology (ESO) Metastatic Breast Cancer Task Force in cooperation with the EBCC (European Breast Cancer Conference), ESMO (European Society of Medical Oncology) and the American $\mathrm{JNCl}$ (Journal of the National Cancer Institute). The main focus of the ABC1 Conference was metastatic breast cancer (stage IV). The ABC1 consensus is based on the vote of 33 breast cancer experts from different countries and has been specified as a guideline for therapeutic practice by the German expert group. It is the objective of the $A B C 1$ consensus as well as of the German comments to provide an internationally standardized and evidence-based foundation for qualified decision-making in the treatment of metastatic breast cancer.

*Writing committee

\section{Schlüsselwörter}

ABC1-Konsensuskonferenz - Metastasiertes Mammakarzinom, Diagnose und Staging, Behandlung . Tumormarker - Metastasen, Biopsie - Chemotherapie . Endokrine Therapie - Anti-HER2-Therapie . Palliativbehandlung

\section{Zusammenfassung}

Eine Arbeitsgruppe deutscher Brustkrebsexperten hat die Abstimmungsergebnisse der ersten internationalen Konsensuskonferenz zur Diagnostik und Behandlung des fortgeschrittenen Mammakarzinoms kommentiert (ABC1). $A B C 1$ steht für "Advanced Breast Cancer First Consensus". Die ABC1-Konferenz ist eine Initiative der Taskforce Metastasiertes Mammakarzinom der European School of Oncology (ESO) in Kooperation mit EBCC (European Breast Cancer Conference), ESMO (European Society of Medical Oncology) und dem US-amerikanischen JNCI (Journal of the National Cancer Institute). Inhaltlicher Schwerpunkt der ABC1-Konferenz war eine Bestandsaufnahme der modernen Therapiestrategien bei Patientinnen mit metastasiertem Mammakarzinom (Stadium IV). Der ABC1-Konsens basiert auf dem Abstimmungsergebnis von 33 Brustkrebsexperten aus verschiedenen Ländern. Dieses wurde durch die deutsche Expertengruppe für den Therapiealltag in Deutschland konkretisiert. Ziel des $A B C 1-K o n s e n s$ sowie der deutschen Kommentierung ist es, eine evidenzbasierte Grundlage für die Therapieentscheidung in der metastasierten Therapiesituation bei Patientinnen mit Mammakarzinom zu schaffen.

\begin{tabular}{ll}
\hline KARGER & ( ) 2012 S. Karger GmbH, Freiburg \\
1661-3791/12/0071-0052\$38.00/0 \\
Fax+49 7614520714 \\
$\begin{array}{l}\text { Information@Karger.de } \\
\text { www.karger.com }\end{array}$ & $\begin{array}{l}\text { Accessible online at: } \\
\text { www.karger.com/brc }\end{array}$
\end{tabular}

Prof. Dr. med. Christoph Thomssen

Klinik und Poliklinik für Gynäkologie

Martin-Luther-Universität Halle-Wittenberg

Ernst-Grube-Strasse 40, 06097 Halle / Saale, Germany

Tel. +49 345 557-1847, Fax -1501

christoph.thomssen@medizin.uni-halle.de 


\section{Introduction}

The first consensus conference for advanced breast cancer ABC1 (= Advanced Breast Cancer First Consensus Conference) held in Lisbon 2011 was organized by the Metastatic Breast Cancer Task Force of the European School of Oncology (ESO) in cooperation with the EBCC (European Breast Cancer Conference), ESMO (European Society of Medical Oncology) and the American JNCI (Journal of the National Cancer Institute). The $\mathrm{ABC} 1$ consensus is based on the procedure of the St. Gallen consensus for early breast cancer which is held for more than twenty years and has become globally recognized.

It is the objective of the $\mathrm{ABC} 1$ consensus to standardize care for patients with advanced breast cancer $(A B C)$ in order to provide an evidence-based therapy to all patients presenting with $\mathrm{ABC}$. The $\mathrm{ABC} 1$ consensus was focussed on metastatic breast cancer (stage IV). The next consensus meeting (ABC2) will focus on locally advanced breast cancer. Therapeutic decisions in metastatic breast cancer are highly influenced by individual criteria resulting from different previous therapies, disease localization and burden as well as an increase in the number of patients with co-morbidities. In light of these facts, the panellists deliberately did not vote on individual substances or therapy sequences.

The ABC1 panel consisted of 33 experts from 15 countries, including 2 representatives from Germany. The statements to be agreed on had been prepared in advance and were openly discussed by the panellists who could vote either 'yes' (agreement), 'no' (rejection) or 'abstention'. Even though the panellists were urged to vote based on published, evidence-based data as defined by the 'clinical practice guidelines' (CPG), the recommendations are ultimately based on opinions from experts representing different fields and countries with different health care systems and resources. It is therefore helpful to comment on the results of the consensus from a German perspective in order to integrate the recommendations in routine care in Germany.

\section{General Treatment Aspects}

All ABC1 panellists (100\%) agreed that the treatment of metastatic breast cancer is complex. To be able to provide adequate treatment, they called upon an interdisciplinary team of highly experienced experts in the treatment of patients with metastatic breast cancer for concerted therapeutic decision-making. Involvement of oncological experts with the following specializations in the treatment team was considered as crucial: medical, radiotherapeutical and surgical/ gynaecological oncologists, imaging experts, pathologists and specialists in the field of palliative and supportive care of tumour patients, including a psycho-oncologist, social aid specialists and a nursing team specialized in breast cancer care. In a separate vote, the $\mathrm{ABC} 1$ panellists recommended that tailored psychosocial supportive and symptom based care should be integrated into the treatment of metastatic disease at an early stage, beginning ideally at the time of diagnosis.

The German experts absolutely agree on both voting results. They refer to the structure of German Breast Centres where these requirements are already established. The treatment of breast cancer patients in certified breast centres is recommended because they provide the framework for quality-assured treatment due to a stringent certification processes and yearly quality control.

\section{Involving Patients in Therapeutic Decisions}

The ABC1 panel agreed that the disease itself and treatment goals should be discussed with the patient before commencing with therapy. This should be done in a comprehensible vocabulary and with the necessary respect for privacy. The patient should be actively involved in therapeutic decision-making. Ideally, patients should be given the most important information in written form. Patients should be informed that there is most likely no curative treatment for the disease. The treatment goals should be discussed with patients in light of this fact. In consultation with the patient, close relatives or other confidants who provide personal and emotional support should be involved in the therapeutic decision-making. The German experts fully agree and emphasize the importance for patients to be fully informed of their most likely incurable situation and that other treatment goals such as stabilizing the disease, reducing symptoms, maintaining quality of life and integration into the social environment are therefore more important.

All ABC1 panellists (100\%) agreed that, in view of only a few evidence-based standards, patients with metastatic breast cancer should preferably be treated within the framework of controlled clinical studies. The ABC1 panel alerted to the rising costs of therapy outside of clinical studies. Nevertheless, the panel stated clearly that therapeutic decisions should not be based on financial considerations but in all instances on the well-being of the patient, her expectations and quality of life, and mutually defined treatment goals. The German expert group absolutely agrees with each of these points.

\section{Systematic Data Documentation}

The majority of the $\mathrm{ABC} 1$ panellists (94\%) were in favour of a systematic documentation of data pertaining to metastatic breast cancer in order to document the effects of disease burden and the resulting symptoms on the patient's quality of life. In the framework of clinical studies, these data should be collected and evaluated systematically in order to include them in future therapeutic decision-making. The German experts consider systematic data collection a desirable approach for treatment optimization of breast cancer patients. However, they described the implementation as difficult. At the 
moment there are no resources available for this task; they should be made available.

\section{Diagnosis and Staging}

\section{Focus on Imaging}

There was a passionate debate about the minimum requirements for an adequate staging procedure in the diagnostic work up of breast cancer metastases. Two thirds (67\%) of the $\mathrm{ABC} 1$ panellists agreed that patient history and clinical examination and - based on the disease and symptoms - complete haematological and biochemical laboratory testing as well as the use of imaging techniques (chest, abdomen, bones) are necessary staging measures. From a German perspective, patient history and clinical examination, complemented by imaging techniques are the most important staging measures (level of evicence (LoE) 1C). Baseline documentation is essential for making any further therapeutic decisions. From the German perspective, chest $\mathrm{X}$-ray is mandatory. If clinical decisions would significantly be influenced, additionally computed tomography of the thorax and abdomen can be performed. Only in well-defined cases PET-CT may be an alternative. However, basically PET-CT is only meaningful to exclude further metastases in patients with a potentially curative option e.g. in case of oligometastasis.

The ABC1 panel did not give any recommendation on the use of imaging techniques in cases of suspected brain metastases in patients with HER2 overexpression or triple negative breast cancer (TNBC) patients. The German expert group agrees: Although the incidence of brain metastases in these patient groups is significantly higher, we lack evidence based clinical data that suggest therapeutic relevance. Therefore, patient history and clinical examination are the decisive diagnostic measures also for patients with HER2 overexpression or triple negative breast cancer.

\section{The Importance of Tumour Markers}

The ABC1 panellists agreed that the clinical relevance of tumour markers is limited. Their clinical relevance has not been sufficiently validated neither for diagnosis nor for follow-up. However, tumour markers might be a useful supplement to monitor response to treatment, especially in patients with non-measurable disease even though they have not been validated as the sole basis for therapeutic decision-making. The German expert group basically agrees to this, but points out that there are clinical situations in which metastatic disease cannot be detected in follow-up by clinical examination or imaging techniques alone. This can be the case in osteoblastic metastases, for instance. In this case, the treating physician can only rely on tumour markers as the basis for his/ her decision. According to the German experts, there are rare cases of discordant development between tumour markers and disease progression.

\section{Treatment Monitoring}

The majority of the $\mathrm{ABC} 1$ panellists $(81 \%)$ recommend reevaluations of treatment response at regular intervals - every 2-4 months during endocrine therapy and after 2-4 cycles during chemotherapy - depending on the dynamics of the disease as well as metastatic spread and location. The use of imaging techniques is only recommended for the marker lesion, if indicated. In an indolent course of disease, longer intervals may also be justified. If clinical suspicion indicates a renewed disease progression, an immediate examination should be carried out - irrespective of the agreed date for the next follow-up examination. The German expert group agrees. It points out that patient history and physical examination have the highest priority and should not be replaced by imaging techniques.

\section{Biopsy of Metastases}

A hot topic was the indication to re-biopsy a newly diagnosed metastatic lesion. Indications for biopsy are confirmation of the diagnosis as well as the re-evaluation of hormone receptor (HR) status and HER 2 expression. In up to $20 \%$ of metastatic patients, tumour biology changes when compared with the primary tumour. The $\mathrm{ABC} 1$ panellists recommend re-biopsy of the metastastatic site for both, confirmation of the diagnosis $(96 \%)$ and re-evaluation of HR and HER2 status (90\%). The German expert group agrees in full with both recommendations referring to the fact that knowledge of tumour biology is a decisive criterion for implementing differentiated therapy. With LoE 1C, the German expert group considered the evidence level to be correspondingly high and went beyond the evidence level of the $\mathrm{ABC} 1$ panellists ( $\mathrm{LoE} 2 \mathrm{C}$ ).

There was agreement between the majority of the $\mathrm{ABC} 1$ panellists $(87 \%)$ and the German experts that targeted therapy (endocrine or anti-HER2 targeted) should be given in cases of discordance between the biology of the primary tumour and metastases if receptors are tested positive in at least one biopsy. This recommendation is being made despite insufficient data since a corresponding controlled clinical trial cannot be expected soon. The German experts are aware of the existing uncertainty.

\section{Therapeutic Decisions}

The great heterogeneity in the treatment of metastatic disease is a fundamental reason for the lack of standardized therapy. There was total agreement $(100 \%)$ among the ABC1 panellists and the German expert group concerning the question of which factors are relevant for therapeutic decision-making and must be considered in all cases. In addition to this, the German experts recommend considering the toxicities of previous and planned therapies in making therapeutic decisions. Moreover, assessment of following factors relevant to treatment is suggested: 
1) HR/HER2 status,

2) menopausal status,

3) disease dynamics/symptom control,

4) performance status, biological age, co-morbidities (incl. organ dysfunction) and co-medication, adverse reactions,

5) previous cancer therapy, disease-free interval,

6) tumour burden (localisation and metastatic spread),

7) socio-economic and psychological factors,

8) available therapy options (i.e. country-specific).

The German experts emphasize that patients should be involved in therapeutic decision-making, and that available therapy options depending on the medical factors should be discussed with the patient. Ultimately, the patient's wishes are determining in making therapeutic decisions.

The German expert group agrees with the ABC1 consensus recommendation $(93 \%)$ that the age of a patient is not per se a reason to forego an effective treatment. However, the German experts pointed out that individual life expectancy, which is influenced by age and possible co-morbidities, should be included in therapeutic decisions.

\section{Oligometastatic Disease}

Patients with limited metastatic spread represent a small but important group. These patients may have a chance of complete remission or of long-term survival, which is why a multimodal treatment concept should be considered on a case-bycase basis. $96 \%$ of the $\mathrm{ABC} 1$ panellists agreed upon this. The German expert group supported this decision, considering the potential for curative treatment in these rare situations. Therefore, patients with singular metastases should be discussed by an interdisciplinary team (tumour board). These patients should be informed about the option of combined, multimodal treatment concepts including surgery of metastases, targeted radiation and adequate systemic therapy.

\section{Resection of the Primary Tumour in Stage IV Disease}

The ABC1 panellists (100\%) agreed that the primary tumour in metastatic disease (stage IV) can be resected as long as this is feasible and the primary tumour will be a subject of R0 resection. The German experts agreed and even recommended the resection of the primary tumour, as long as contraindications are absent and $\mathrm{R} 0$ resection can be expected, since retrospective data suggest that $\mathrm{R} 0$ resection has a positive effect on the further course of the disease even in stage IV breast cancer.

\section{Endocrine Therapy}

There was decisive evidence with a correspondingly high consensus among the $\mathrm{ABC} 1$ panellists and agreement on the side of the German expert group on the recommendations for endocrine therapy of patients with metastatic breast cancer. Endocrine treatment is the preferred therapy for patients with a positive hormone receptor (HR) status. This also applies in cases of visceral metastases. Exceptions calling for primary
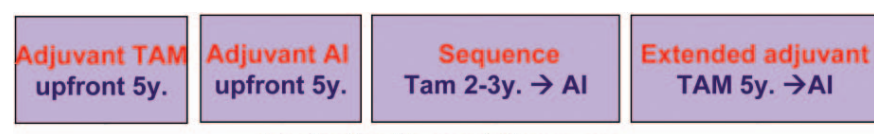

End of adjuvant therapy

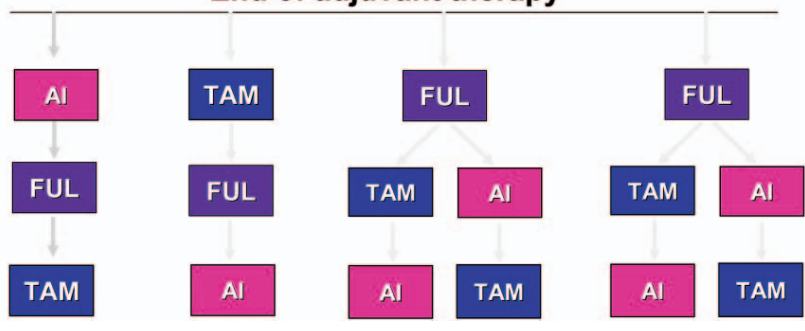

Fig. 1. Endocrine therapy options in advanced breast cancer depending on previous adjuvant therapy [3]. TAM = tamoxifen, $\mathrm{AI}=$ aromatase inhibitor, FUL = fulvestrant, $\mathrm{y}=$ years

chemotherapy are proven endocrine resistance as well as rapidly progressing disease which necessitates prompt treatment response. The German expert group points out that this preference of endocrine treatment only applies to HER2 negative patients.

In pre-menopausal patients with positive HR status, the suppression of ovarian function in combination with additional endocrine treatment - preferably tamoxifen - is the standard of care. According to the ABC1 consensus, the application of an aromatase inhibitor instead of tamoxifen constitutes an option. The German expert group emphasizes that pre-menopausal patients may only be treated with aromatase inhibitors in combination with ovarian suppression (LHRH agonist).

In post-menopausal patients with a positive HR status, aromatase inhibitors are the preferred first-line endocrine therapy. For selected patients, tamoxifen remains a valid treatment option. The German expert group specifies that previous adjuvant therapy should be taken into consideration when making therapeutic decisions. This also applies to the question of whether or not an aromatase inhibitor should be recommended in general and which aromatase inhibitor should be recommended specifically. A reinduction of previous endocrine therapy may be considered if a patient has been stable and off therapy for a sufficiently long time. (fig. 1).

Currently, optimal therapy following aromatase inhibitor therapy is not sufficiently defined. According to the ABC1 panellists, the options currently available for a further treatment are non-cross-acting aromatase inhibitors, tamoxifen, fulvestrant and megestrol acetate. The German expert group agrees with this statement. The group recommends a noncross-acting aromatase inhibitor as the primary therapy option. Moreover, the German experts point out a possible combination of endocrine therapy and the mTOR inhibitor everolimus, an option which has been shown to be effective in the current evaluation of clinical trials $[1,2]$.

The majority of the ABC1 panellists (53\%) with an abstention of $20 \%$ were not in favour of considering the use of the mTOR inhibitor everolimus combined with tamoxifen (TAM- 
RAD trial) or the steroidal aromatase inhibitor exemestane (BOLERO 2 trial) for patients with acquired endocrine resistance at this point in time. $77 \%$ of the panellists were against definite recommendations for the combined treatment. A simple majority (48\%) of the $\mathrm{ABC} 1$ panellists agreed that further study data are needed before respective recommendations for the clinical use of everolimus in combination with tamoxifen or aromatase inhibitors can be made. The German experts agree with this, although they deem the call for further trials unrealistic. Therefore, the German experts recommend waiting for full publication of the results of the two relevant trails - TAMRAD and BOLERO 2 - in the New England Journal of Medicine [1].

There are no current randomized clinical trials investigating further endocrine treatment options for patients with a positive HR status, who had primarily received chemotherapy. Nevertheless the majority of $\mathrm{ABC} 1$ panellists were in favour of further endocrine treatment as maintenance therapy. The German experts agree since this therapeutic concept is well established as common practice and has proven successful. The ABC1 panellists (100\%) and the German experts agreed that simultaneous administration of chemotherapy and endocrine treatment is not recommended. A survival benefit has not been shown. The German experts point out that potentially antagonistic effects of both treatment approaches cannot be excluded. The recommendation does not apply to future combinations of potential new drugs. These should be evaluated separately in future clinical trials.

\section{Anti-HER2 Targeted Therapy}

Patients with HER2-positive metastatic breast cancer should be offered an anti-HER2 targeted therapy at an early stage as part of the first-line therapy as long as no contraindications apply. $91 \%$ of the $\mathrm{ABC} 1$ panellists were in agreement with this statement, which was supported by the German expert group as well. This recommendation is in accordance with the available data and the current recommendation of the AGO Mamma (www.ago-online.org). The German experts point out that for the proven survival benefit it is principally recommended to combine anti-HER2 targeted therapy with chemotherapy. If chemotherapy is not an option for patients with a positive HR status and HER2 overexpression, the anti-HER2 targeted therapy should be combined with an endocrine therapy. In comparison with endocrine therapy alone, controlled clinical trials have shown a significant prolongation of progression-free survival, though not yet a survival benefit for the combination of anti-HER2 and endocrine therapy. The German expert group principally agrees, but they point out that the combination of anti-HER2 targeted therapy and an aromatase inhibitor can be considered for patients with little or no symptoms of metastatic disease or slow progression of disease. Both options - the combination of anti-HER2 therapy with endocrine or chemotherapy - should be discussed with the patient.
Treatment Beyond Progression (TBP) while on Anti-HER2

\section{Targeted Therapy}

In case of progressive disease under a combination treatment of anti-HER2 targeted therapy plus chemotherapy, the ABC1 panellists (97\%) recommend switching to an alternative combination of anti-HER2 targeted therapy plus endocrine or chemotherapy in order to maintain the always mandatory HER2 blockade. The ideal length of anti-HER2 targeted treatment is as yet unknown. The German experts agree with both statements. The recommendation to continue with an alternative anti-HER2 therapy regimen is in accordance with common clinical practice. The $\mathrm{ABC} 1$ panellists and the German expert group agreed that the combination of endocrine therapy (for HR+ patients) with anti-HER2 therapy as well as continuing treatment with trastuzumab and a chemotherapy alternative (TBP) but also switching to lapatinib in combination with capecitabine are valid options.

Treatment for patients with HER2-positive metastatic disease who have received anti-HER2 directed therapy in the adjuvant setting is still unclear. The $\mathrm{ABC} 1$ panellists agreed that anti-HER2 targeted therapy should be continued in firstline therapy of metastatic disease. Which drugs or regimens should be used depends largely on the availability in individual countries. In addition, further treatment should be based on the duration of the time interval between therapies as well as previous adjuvant therapies.

The German experts recommend a re-induction with trastuzumab if at least 2 years have elapsed since the previous therapy. Should less than 2 years have elapsed since prior therapy switching to lapatinib/capecitabine is recommended. The ABC1 panellists (100\%) and the German expert group agree that clinical studies addressing this situation are necessary.

The dual HER2 blockade shows promising results in clinical trial settings. Although still lacking approval, the majority of the $\mathrm{ABC} 1$ panellists $(83 \%)$ were in favour of the dual HER2 blockade (trastuzumab/lapatinib) as a useful therapeutic option in cases of trastuzumab resistance. The German experts consider the dual HER2 blockade a biologically and clinically meaningful therapeutic option; its use should principally be advocated and, since approval is still pending, well documented and justified. When to recommend this therapy regime is still unclear, since controlled clinical trails have proven efficacy only after trastuzumab resistance and not after progression on lapatinib/capecitabine therapy.

\section{Focus on Chemotherapy}

\section{No Standardized Therapy Algorithm}

If chemotherapy is indicated in metastatic situations, the $\mathrm{ABC} 1$ consensus prefers to recommend sequential monotherapy. Polychemotherapy should be an option for patients with rapid tumour progression, acute life-threatening visceral
56

Breast Care 2012;7:52-59
Thomssen/Marschner/Untch/Decker/ Hegewisch-Becker/Jackisch/Janni/Lück/ von Minckwitz/Scharl/Schneeweiss/Tesch/ Welt/Harbeck 
metastases, for patients requiring rapid disease control or symptom mitigation. This is in agreement with current AGO recommendations, thus the German expert group agrees totally.

Due to the heterogeneity of the disease, there is no definitive therapeutic algorithm for the use of cytotoxic substances in the treatment of metastatic breast cancer. The ABC1 panellists and the German expert group agree that individual substances should be applied according to available evidence and dependent on the individual treatment situation. Detailed recommendations from a German perspective are given in the current AGO recommendations (www.ago-online.org).

The German expert group points out that it is important to discuss treatment options and to define treatment goals with the patient in order to make a mutual therapy decision. The current AGO recommendations include a list of all substances that may be used in the course of the first 3 therapy lines in metastatic situations. Taking into consideration possible (neo) adjuvant pretreatment, anthracyclines and taxanes are substances of first choice. Typical second choice compounds are vinorelbine or capecitabine and, for heavily pretreated patients, eribulin. Should a polychemotherapy be indicated, the German expert group - in agreement with the AGO recommendations - prefers to recommend the following combinations: anthracycline/taxane or taxane/gemcitabine in the case of adjuvant anthracycline therapy. Combinations of second choice are: taxane/capecitabine and liposomal anthracycline/ cyclophosphamide.

\section{Duration of Chemotherapy}

The ABC1 panellists (96\%) agreed that the duration of therapy and the number of therapy lines should depend on the individual situation. $72 \%$ of panellists were in favour of continuing treatment until either progression or occurrence of unacceptable side effects. The German experts restrict this statement only to monotherapy. A polychemotherapy should be given for a maximum of 18-24 weeks or until the best treatment response is achieved. In addition, the German expert group points to the option of intermittent treatment (socalled 'drug holidays'), which was not discussed by the ABC1 panellists.

The ABC1 panellists gave no general recommendation for first-line therapy with bevacizumab in combination with taxanes. The addition of bevacizumab to first-line taxane chemotherapy has shown an advantage of 5 months in progression-free survival in controlled clinical trials but no improvement in overall survival. Due to the lack of predictive factors and in light of the PFS advantage mentioned above, $74 \%$ of the $\mathrm{ABC} 1$ panellists voted in favour of considering the additional administration of bevacizumab a possible option in individual cases. The German expert group points out that bevacizumab has been approved in Europe in combination with taxanes or capecitabine for first-line therapy of HER2-negative metastatic breast cancer. The current
AGO recommendations contain information on bevacizumab therapy options.

\section{Treatment of Specific Metastatic Situations}

\section{Bone Metastases}

The majority of $\mathrm{ABC} 1$ panellists (96\%) supported the view that patients with bone metastases should receive bone-specific drugs - a bisphosphonate or denosumab - in addition to systemic tumour therapy. The German expert group agrees. A ranking of these substances is not recommended.

The ABC1 panellists agreed on an imaging procedure in cases of symptomatic bone metastases in order to identify impending or actual fractures. If no fracture can be identified, radiotherapy is recommended. In the case of increased fracture risk or an existing fracture, consultation with a surgeon specialized in bone surgery and surgical stabilization should be considered. In case of surgery, postoperative radiotherapy is recommended. The German expert group agrees with this recommendation and emphasizes that patients with painful bone metastases should generally be discussed in an interdisciplinary board (tumour board). If bone metastases threaten bone stability, particularly in the spine, an experienced orthopaedic surgeon, traumatologist or neurosurgeon should be consulted immediately.

\section{Neurological Symptoms}

All ABC1 panellists (100\%) agreed that spinal cord compression must be considered as a possible cause of neurological complaints and deficits and that this emergency situation should elicit an immediate response. All potentially affected areas, including neighbouring areas of the spine, must undergo thorough radiological examination. Magnetic resonance tomography (MRT) is the standard of care. A traumatologist, neurosurgeon or orthopaedic surgeon should be consulted immediately. In cases where comorbidity does not permit stabilizing or de-compressing surgery with subsequent irradiation, emergency irradiation is the therapy of choice. The German expert group agrees. Because of the risk of paraplegia, it is absolutely necessary to arrange immediate consultation of an orthopaedic surgeon, traumatologist or neurosurgeon as well as a radiotherapist.

\section{Brain Metastases}

The German expert group agrees with the majority of the ABC1 panellists $(92 \%)$ that patients with singular or few small, potentially operable brain metastases can undergo surgery or stereotactic irradiation. The majority of panellists $(72 \%)$ voted in favour of subsequent whole brain radiotherapy (WBRT). From a German perspective, surgical resection, if possible, is the primary therapeutic option. The German expert group specifies that additional WBRT should be generously recommended, and refers to the current AGO 
recommendations ( $\mathrm{AGO}++$, www.ago-online.org). The additional WBRT does not achieve a significant survival benefit, but can prevent intracranial recurrences and contribute to a prolonged maintenance of neurological function. This should be discussed with the patient. The German experts add that corticosteroids should be administered to patients receiving whole brain or stereotactic irradiation. There was no further discussion among ABC1 panellists pertaining to important questions such as whether or not to recommend capecitabine/lapatinib for patients with HER2positive metastatic breast cancer as an alternative to CNS irradiation or general treatment approaches for leptomeningeal carcinomatosis.

\section{Palliative Care}

The $\mathrm{ABC} 1$ panellists and the German expert group agree that palliative medical care provided by experts plays an important role in the treatment of metastatic disease. Adequate palliative care should be integrated into planned therapy right from the start to guarantee optimal safety and tolerance.

The German expert group expands on these two general $\mathrm{ABC} 1$ consensus statements: Important aspects of palliative treatment are antiemesis, analgesia, bisphosphonate/denosumab therapy, physiotherapy and optional psycho-oncological therapy. The German experts recommend caution concerning blood transfusions and erythropoietin administration. Eythropoietin should only be administered in individual cases, with very low $\mathrm{Hb}$ values $(<10 \mathrm{~g} / \mathrm{dl})$. In purely palliative treatment situations, therapy modifications such as drug holidays or dose reductions should be favoured over the administration of growth factors in case of therapy-induced side effects.

The German expert group emphasizes that palliative care should play an integral role in the oncological therapy of metastatic breast cancer and must be adapted to the medical condition and needs of each individual patient. In individual cases, particularly in the final stage of disease, specialized palliative medical therapy (SPV) should complement general palliative care. The possibilities offered by palliative wards in certified breast centres should be utilized.

The ABC1 panellists and the German expert group absolutely agree that adequate pain therapy (e.g. morphine) for optimal pain control is a necessary prerequisite for adequate palliative care of all tumour patients. The German experts specify that adequate pain medication according to the WHO pain relief scale constitutes standard therapy. In Germany this does not pose a problem, as access to opioids is regulated by law.

\section{Respecting Patient's Preferences}

In the final stage of disease patient's preferences are of particular importance. These should be discussed with her and her relatives at an early stage, preferentially before but at the latest when the disease can no longer be controlled adequately. The majority of the ABC1 panellists (96\%) and the German expert group agree on this point. The German experts point out that this procedure is considered standard of care in Germany. In the final stage of disease, purely palliative care (meaning 'best supportive care') is always an alternative to anti-tumour therapy and should be discussed with the patient.

\section{Male Breast Cancer}

Male breast cancer is rare and usually hormone-sensitive (positive HR status). Therefore, therapy of choice is endocrine treatment, preferably with tamoxifen, as long as there is no suspicion of endocrine resistance or aggressive disease progression calling for a rapid response. In case of progression on tamoxifen, aromatase inhibitors are a justifiable therapeutic option. The ABC1 panellists and the German expert group agreed on this point. However, it is unclear whether aromatase inhibitor therapy should be accompanied by an LHRH agonist. A marginal majority of the ABC1 panellists $(58 \%)$ were in favour of concurrent LHRH agonist therapy, whereas almost one third (29\%) abstained from voting. The German expert group points out that there are no definitive data to support this combination, which is why the addition of a LHRH agonists to aromatase inhibitors cannot be recommended.

The German experts emphasize that it is necessary to collect more data on male breast cancer. Until then, male breast cancer patients should be treated in the same manner as female breast cancer patients. This applies to chemotherapy and anti-HER2 targeted treatment recommendations as well.

\section{Summary}

The first consensus meeting in Lisbon focusing on advanced breast cancer $(\mathrm{ABC} 1)$ has successfully discussed and voted on therapeutic guidelines for patients with metastatic breast disease. In many aspects, these are in accordance with the evidence-based recommendations of the German AGO Breast committee (www.ago-online.de).

The German task force considers the $\mathrm{ABC} 1$ to be an important and highly successful international initiative. The second Consensus Conference on Advanced Breast Cancer (ABC2) will take place November 7-9, 2012 in Lisbon focussing an locally advanced and recurrent breast cancer.

\section{Disclosure Statement}

The meeting of the German experts was sponsored by Celgene. The manuscript was drafted by Birgit-Kristin Pohlmann.
Thomssen/Marschner/Untch/Decker/ Hegewisch-Becker/Jackisch/Janni/Lück/ von Minckwitz/Scharl/Schneeweiss/Tesch/ Welt/Harbeck 


\section{References}

1 Baselga J, Campone M, Piccart M, Burris HA, Rugo HS, Sahmoud T, Noguchi S, Gnant M, Pritchard KI, Lebrun F, Beck JT, Ito Y, Yardley D, Deleu I, Perez A, Bachelot T, Vittori L, Xu Z, Mukhopadhyay P, Lebwohl D, Hortobagyi GN. Everolimus in Postmenopausal Hormone-Receptor-Positive Advanced Breast Cancer. N Engl J Med. 2011 Dec 7. [Epub ahead of print]
2 Hortobagyi GN, Piccart M, Rugo H, Burris H, Campone M, Noguchi, Gnant M, Pritchard KI, Vittori L, Mukhopadhyay P, Sahmoud T, Lebwohl D, Baselga J. Everolimus for postmenopausal women with advanced breast cancer: updated results of the BOLERO-2 phase III trial. S3-7. San Antonio Breast Cancer Symposium 2011.
3 Harbeck N: ABC-1 Conference November 2011, oral presentation. 


\section{Erratum}

In the article

ABC1 Consensus Conference - a German Perspective. First International Consensus Conference on Advanced Breast Cancer (ABC1), Lisbon, November 5, 2011 (Breast Care 2012;7:52-59 DOI: 10.1159/000336049)

there was a mistake in the introduction: instead of 'The ABC1 Conference is an initiative of the European School of Oncology (ESO) Metastatic Breast Cancer Task Force in cooperation with the EBCC (European Breast Cancer Conference), ESMO (European Society of Medical Oncology) and the American JNCI (Journal of the National Cancer Institute).' it should read 'The ABC1 Conference is an initiative of the European School of Oncology (ESO) following a 6-year work from the ESO-MBC Task Force.' 